\title{
Interferon-Induced Transmembrane Protein: A Moonlighting Protein Against SARS-CoV-2 Infection or in Support of Invasive Ductal Breast Carcinoma?
}

\section{Rinki Minakshi}

Department of Microbiology, Swami Shraddhanand College, Delhi University, India.

\begin{abstract}
The interferon-induced transmembrane proteins (IFITMs), widely acting against invading viruses are ubiquitously expressed on the cellular membranes, were previously known for their prominent role in tumorigenesis. Studies productively showed that the entry restriction on SARS-CoV spike glycoprotein agreeably involved the action of frontier IFITM1, 2 and 3. On the contrary, overexpression of IFITM3 has been reported in Invasive ductal breast carcinoma (IDC) tissue specimens where lentivirus-delivered shRNA resulted in targeted silencing of IFITM3 mRNA expression. Despite acting protective against virus infection, expression of IFITM favors cancer migration as seen in IDC. The existence of such a phenomenon wherein a choice is made by the selection pressure on IFITM allele frequency in human population between opposing roles of the protein, needs to be untangled.
\end{abstract}

Keywords: Breast cancer-Covid-19- Interferon-induced transmembrane protein-Invasive ductal breast carcinoma

\section{Introduction}

The innate immunity, being an autonomous cellular arsenal against invading viruses, has strategically evolved its surveillance power as well as effector functions. The potent immune mediator, interferon (IFN), is widely secreted against invading viruses. The whole plethora of genes evoked in response to the secreted IFNs comprises of IFN-stimulated genes (ISGs), which function primarily by imposing modulation on multiple stages of viral replication event [1]. The interferon-induced transmembrane proteins (IFITMs), labelled as a moonlighting protein, are one such ubiquitously expressed ISGs, on the cellular membranes, that were previously known for their prominent role in tumorigenesis. Widened functional studies have extended striking role of IFITMs, wherein IFITMs not only interrupt host-virus membrane fusion but also impede infectious virion production in cell culture [2]. Normally, cells show basal expression levels of IFITMs, which significantly see an upsurge in their levels during virus infection [3]. As witnessed in studies using siRNA, IFITM1, -2 and -3 have acted by inhibiting infection establishment in influenza A virus (IAV), West Nile virus, dengue virus,
Marburg virus, Ebola virus, HIV-1 and SARS-CoV [4]. The mechanistic details of this inhibition are still in its juvenile stage, but studies have emphasized that IFITM proteins inhibit enveloped viruses [5].

Studies productively showed that the entry restriction on SARS-CoV spike glycoprotein agreeably involved action of frontier IFITM1, 2 and 3 rather than concomitant ACE2 downregulation. The shRNA targeting of IFITM1 markedly enhanced SARS-CoV spike mediated entry without altering expression of ACE2. Moreover, the exploitation of other host factors apart from the primary receptor of the virus, cannot be overlooked in this mechanism [6]. Congruently, MERS-CoV entry has also been shown to be inhibited by IFITM proteins [5]. On the contrary, human coronavirus-OC43 (HCoV-OC43), the etiological agent of common cold, seizes IFITM proteins for their entry thereby proving the predictability of evasion tactics against IFITM restrictions by viruses [7].

Invasive ductal breast carcinoma (IDC), which accounts for approximately $80 \%$ of breast cancer cases, is initiated by a set of multifaceted pathogenesis

\section{Corresponding Author:}

Dr. Rinki Minakshi

Department of Microbiology, Swami Shraddhanand College, Delhi University, India.

Email: minakshi4050@gmail.com 
mechanism and gene mutations. Overexpression of IFITM3 has been reported in IDC tissue specimens where lentivirus-delivered shRNA resulted in targeted silencing of IFITM3 mRNA expression. The study further proved that the knockdown of IFITM3 led to significant reduction in tumor viability [8]. There are several reports on the overexpression of IFITM3 in various tumors like human glioma, colon cancer, esophageal squamous cell carcinoma and hepatocellular carcinoma [9]. Another study showed that IFITM3 ameliorated inflammation and colitis-associated tumorigenesis [9]. On the contrary, IFITM3 overexpression lead to loss of fetus development in mice, which has been correlated with a similar pathology during Zika virus infection [9].

All the aforementioned studies notify about a highly significant aspect of IFITM expression where balance between high and low IFITM levels is evident as frequency of circulating alleles (single nucleotide polymorphism, SNP) in human population. The SNP rs12252T $>$ C (minor IFITM3 allele) was significantly enriched in patients hospitalized during $\mathrm{H} 1 \mathrm{~N} 1$ pandemic (2009) [10]. Moreover, a meta-analysis revealed that IFITM3 rs $12252 \mathrm{~T}>\mathrm{C}$ polymorphism showed substantial association with the risk of developing severe influenza [11]. These SNPs resulted in diminished IFITM3 expression thereby suggesting that selection of fetus development might dominate endurance towards severe virus infection.

The current pandemic imposed by SARS-CoV-2 infection has been puzzling both scientific as well as medical fraternity. Deriving conclusive impetus from aforesaid studies, procuring experimental data on the potential role of IFITMs, might elucidate an aspect of host response during SARS-CoV-2 infection in cancer patients. Despite acting protective against virus infection, expression of IFITM favors cancer migration as seen in IDC. This again points towards the existence of a choice made by the selection pressure on IFITM allele frequency in human population. We emphasize more in-depth study on IFITM SNP and its functional association with cancer as well as SARS-CoV-2 infection, where the mystery behind two opposing effects of IFITM expression in host can be deciphered.

\section{References}

1. Sadler AJ, Williams BRG. Interferon-inducible antiviral effectors. Nature Reviews Immunology. 2008 07;8(7):559568. https://doi.org/10.1038/nri2314

2. Shi G, Schwartz O, Compton AA. More than meets the I: the diverse antiviral and cellular functions of interferon-induced transmembrane proteins. Retrovirology. 2017 Nov 21;14(1). https://doi.org/10.1186/s12977-017-0377-y

3. Siegrist F, Ebeling M, Certa U. The Small Interferon-Induced Transmembrane Genes and Proteins. Journal of Interferon \& Cytokine Research. 2011 01;31(1):183-197. https://doi. org/10.1089/jir.2010.0112

4. Huang I, Bailey CC, Weyer JL, Radoshitzky SR, Becker MM, Chiang JJ, Brass AL, Ahmed AA, Chi X, Dong L, Longobardi LE, Boltz D, Kuhn JH, Elledge SJ, Bavari S, Denison MR, Choe H, Farzan M. Distinct Patterns of IFITM-
Mediated Restriction of Filoviruses, SARS Coronavirus, and Influenza A Virus. Baric RS. PLoS Pathogens. 2011 01 06;7(1):e1001258. https://doi.org/10.1371/journal. ppat. 1001258

5. Wrensch F, Winkler M, Pöhlmann S. IFITM Proteins Inhibit Entry Driven by the MERS-Coronavirus Spike Protein: Evidence for Cholesterol-Independent Mechanisms. Viruses. 201409 26;6(9):3683-3698. https://doi.org/10.3390/ v6093683

6. Zhao X, Sehgal M, Hou Z, Cheng J, Shu S, Wu S, Guo F, Le Marchand SJ, Lin H, Chang J, Guo J. Identification of Residues Controlling Restriction versus Enhancing Activities of IFITM Proteins on Entry of Human Coronaviruses. Williams BRG. Journal of Virology. 2017 Dec 20;92(6). https://doi.org/10.1128/jvi.01535-17

7. Zhao X, Guo F, Liu F, Cuconati A, Chang J, Block TM, Guo J. Interferon induction of IFITM proteins promotes infection by human coronavirus OC43. Proceedings of the National Academy of Sciences. 201404 21;111(18):6756-6761. https://doi.org/10.1073/pnas.1320856111

8. YANG M, GAO H, CHEN P, JIA J, WU S. Knockdown of interferon-induced transmembrane protein 3 expression suppresses breast cancer cell growth and colony formation and affects the cell cycle. Oncology Reports. 201304 26;30(1):171-178. https://doi.org/10.3892/or.2013.2428

9. Protecting fetal development. Paul Kellam, Robin A. Weiss. Science 2019.365(6449):118-9. https://doi.org/10.1126/ science.aay 2054

10. Bailey CC, Zhong G, Huang I, Farzan M. IFITM-Family Proteins: The Cell's First Line of Antiviral Defense. Annual Review of Virology. 2014 Nov 03;1(1):261-283. https://doi. org/10.1146/annurev-virology-031413-085537

11. Xuan Y, Wang LN, Li W, Zi HR, Guo Y, Yan WJ, Chen $\mathrm{XB}$, Wei PM. IFITM3 rs12252 $\mathrm{T}>\mathrm{C}$ polymorphism is associated with the risk of severe influenza: a meta-analysis. Epidemiology and Infection. 201503 17;143(14):2975-2984. https://doi.org/10.1017/s0950268815000278

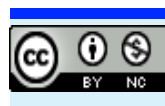

This work is licensed under a Creative Commons AttributionNon Commercial 4.0 International License. 